\title{
Characterization of ZnSe Nanolayers by Spectroscopic Ellipsometry
}

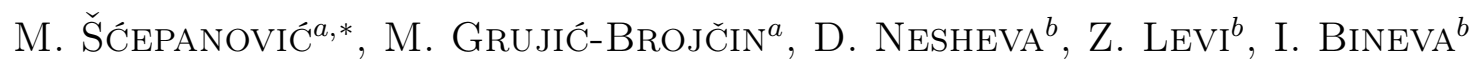 \\ AND Z.V. POPOVIĆ ${ }^{a}$ \\ ${ }^{a}$ Center for Solid State Physics and New Materials, Institute of Physics, Belgrade, Serbia \\ ${ }^{b}$ Institute of Solid State Physics, Bulgarian Academy of Sciences, Sofia, Bulgaria
}

\begin{abstract}
Single layers of ZnSe with thicknesses of 30, 40, 50, 70 and $100 \mathrm{~nm}$ are deposited at room substrate temperature by thermal evaporation of ZnSe powder in vacuum. The layers surface morphology has been investigated by atomic force microscopy. Structural characterization by the Raman scattering measurement revealed the existence of randomly oriented crystalline ZnSe particles in all layers, and the presence of amorphous phase in layers thinner than $100 \mathrm{~nm}$. The ellipsometric measurements were performed in the range from 1.5 to $5 \mathrm{eV}$ at room temperature in air. To interpret the experimental results, the Bruggeman effective medium approximation of dielectric function of ZnSe layers has been used, representing the layers as different mixtures of crystalline ZnSe (c-ZnSe), amorphous ZnSe (a-ZnSe), and voids. The assumption of polycrystalline ZnSe layers modeled as mixture of porous $\mathrm{c}-\mathrm{ZnSe}$ (with volume fraction of voids $\approx 0.17$ ) and a-ZnSe gives the best fit of ellipsometric experimental data. Single layer thicknesses similar to those expected from preparation conditions have been obtained by this fitting procedure. It has been also found that decrease in the layer thickness causes an increase of the volume fraction of a-ZnSe. Thus, c-ZnSe/a-ZnSe ratio, porosity and layer thickness obtained by spectroscopic ellipsometry, provides useful information about crystallinity and micro-/nanostructure of ZnSe nanolayers.
\end{abstract}

PACS numbers: 81.07.-b, 81.15.-z, 81.05.Dz, 78.30.-j, 07.60.Fs, 68.55.-a

\section{Introduction}

Thin nanostructured semiconductor films, II-VI in particular, are promising materials for gas sensor applications ([1] and references therein), due to significantly larger sensing surface than the geometric one. Information concerning phonon propagation in such nanostructures has great technological importance in designing semiconductor optoelectronic and fast electronic devices. Growing interest in $\mathrm{ZnSe}$ low-dimensional structures is also caused by its possible application in effective light-emitting devices with spontaneous or coherent radiation in the green-blue part of the visible spectrum [2-4] or white light emission [5].

The investigation of series of ZnSe single layers deposited at room substrate temperature by thermal evaporation of $\mathrm{ZnSe}$ powder in vacuum is presented in this study. Atomic force microscopy (AFM) is applied to investigate the film surface morphology and structure. The Raman scattering measurements are used to show that as-deposited single layers contain two phases, amorphous and crystalline. Spectroscopic ellipsometry (SE), as a non-contact and nondestructive optical technique for characterization of thin films, is successfully applied for

* corresponding author; e-mail: maja@ipb.ac.rs estimation of thickness and crystallinity of ZnSe single layers. By employing effective medium approximation (EMA) [6], ZnSe single layers are treated as a large-grain polycrystalline material modeled by a mixture of porous crystalline ZnSe (c-ZnSe) and amorphous ZnSe (a-ZnSe) with varying amorphous phase fraction.

\section{Experimental details}

Single layers of ZnSe having thicknesses of 30, 40, 50, 70 , and $100 \mathrm{~nm}$ were deposited on crystalline $p$-Si substrate held at $\approx 25^{\circ} \mathrm{C}$. The layers were produced by physical evaporation of powdered ZnSe (Merck, Suprapure) at a residual pressure of $\approx 3 \times 10^{-4} \mathrm{~Pa}$ from a tantalum crucible located in the bottom of a cylindrical screen (not intentionally heated). The top of the screen is close to the substrates; thus evaporation in a quasi-closed volume is carried out. A preliminary calibrated quartz microbalance system was used to control the nominal layer thickness and deposition rate $\left(V_{\mathrm{d}}=1.5 \mathrm{~nm} / \mathrm{s}\right)$. Step-by-step deposition was carried out during which the substrates were rotated at a rate of 8 turns/min spending only $1 / 12$ part of the turn time over the ZnSe source. Thus, more than 30 "sublayers", with a nominal thickness of $\approx 1.0 \mathrm{~nm}$ in each step, form the respective layer.

The ZnSe layer microstructure was explored using a Nanoscan Atomic Force Microscope in constant frequency regime. An area of $6.64 \times 6.64 \mu \mathrm{m}^{2}$ was scanned 
at various resolutions $(512 \times 512,1024 \times 1024$ and $2048 \times 2048)$.

The Raman measurements were performed in the near-backscattering geometry using the $442 \mathrm{~nm}(2.8 \mathrm{eV})$ line of a $\mathrm{He}-\mathrm{Cd}$ laser as an excitation source, a Jobin-Yvon U1000 double monochromator and photomultiplier as detector.

The ellipsometric spectra of the ZnSe nanopowders were measured using SOPRA GES-5 variable angle ellipsometer in rotating polarizer configuration. The SE data $(\cos (\Delta), \tan (\Psi))$ were collected at room temperature, in the range from 1.5 to $5.0 \mathrm{eV}$ with resolution of $0.03 \mathrm{eV}$ and incidence angle of $70^{\circ}$.

\section{Results and discussion}

A series of ZnSe layers with various thicknesses is investigated by AFM to see if the reduction of layer thickness results in some effects on the layer structure. The surface $3 \mathrm{D}$ image of ZnSe layer with thickness of $70 \mathrm{~nm}$ is shown in Fig. 1. The pits assumed on the basis of the 2D images are clearly seen, and similar results are obtained for the other samples. The rms roughness of the layers varies between 2 and $4 \mathrm{~nm}$ and does not show thickness dependence. The roughness of relaxed films is comparable or smaller than the roughness reported for films of similar thicknesses deposited with atomic layer [5] and chemical bath [7] deposition.

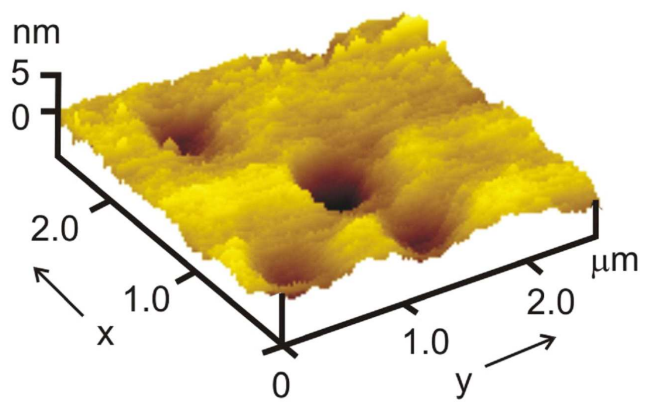

Fig. 1. 3D AFM image of the surface of a ZnSe layer with thickness of $70 \mathrm{~nm}$.

Surface profiles of two samples with different thicknesses (30 and $70 \mathrm{~nm}$ ) along random lines parallel to the $x$-axis of scanning are shown in Fig. 2. Large and short scale variations in the height of surface points are observed. Short scale variations are more pronounced in the thicker layer. The large scale variations may be assigned to the existence of pits and high regions on the film surface, while the small scale ones could be related to the grains with a maximum size of $25-30 \mathrm{~nm}$ in all samples.

The Raman spectra of ZnSe single layers with thicknesses between 30 and $100 \mathrm{~nm}$ are shown in Fig. 3. The observed features in all spectra have been ascribed to multiple optical phonon (1LO to $4 \mathrm{LO}$ ) light scattering and related to the presence of randomly oriented crystalline ZnSe grains in single layers [8]. Relatively large

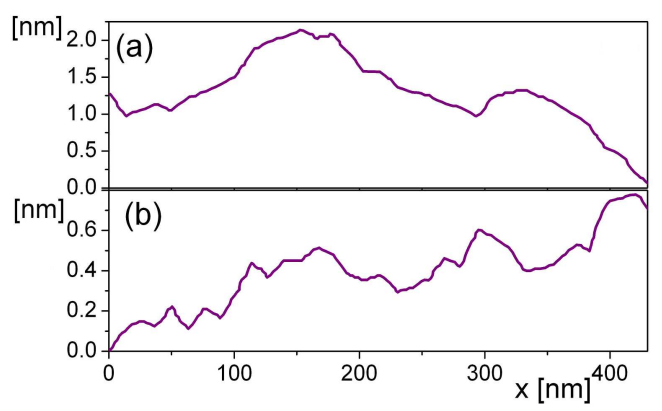

Fig. 2. Surface profile of $30 \mathrm{~nm}$ (a) and $70 \mathrm{~nm}$ (b) thick layers along a random line parallel to the $x$-axis of scanning. The scans were performed at $2048 \times 2048$ steps.

line width of the $1 \mathrm{LO}$ band of $\approx 15 \mathrm{~cm}^{-1}$, in comparison to $\mathrm{ZnSe}$ single crystal value $\left(\approx 6 \mathrm{~cm}^{-1}\right)$, has been related to the lattice distortion in the crystalline grains and to the existence of amorphous phase in the layers thinner than $100 \mathrm{~nm}[8]$.

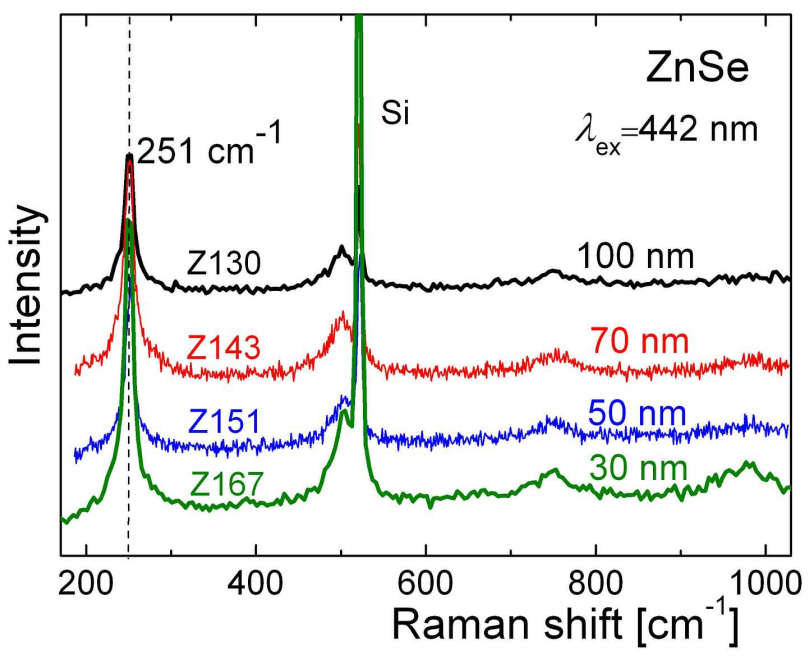

Fig. 3. Raman spectra of a series of ZnSe single layers with four different thicknesses (denoted in the figure) measured using the $442 \mathrm{~nm}$ laser line.

Figure 4 shows the room-temperature ellipsometric data for $\cos (\Delta)$ and $\tan (\Psi)$ of ZnSe single layers. The data analysis requires several steps, the first one is to define a layer structure in the ellipsometric analysis program of SOPRA instrument with the layer thickness as fitting parameter.

It has been reported $[5,7,9]$ that, independently of the deposition method used, the structure of ZnSe layers with $d \leq 100 \mathrm{~nm}$ is a mixture of nanocrystals (with average size of $5-10 \mathrm{~nm}$ ) embedded in an amorphous matrix. Some authors have even mentioned [5] that films deposited on glass or amorphous quartz substrates are completely amorphous. This gives us basis to suggest co-existence of crystalline and amorphous phases in our ZnSe layers having thicknesses between 30 and $100 \mathrm{~nm}$. 


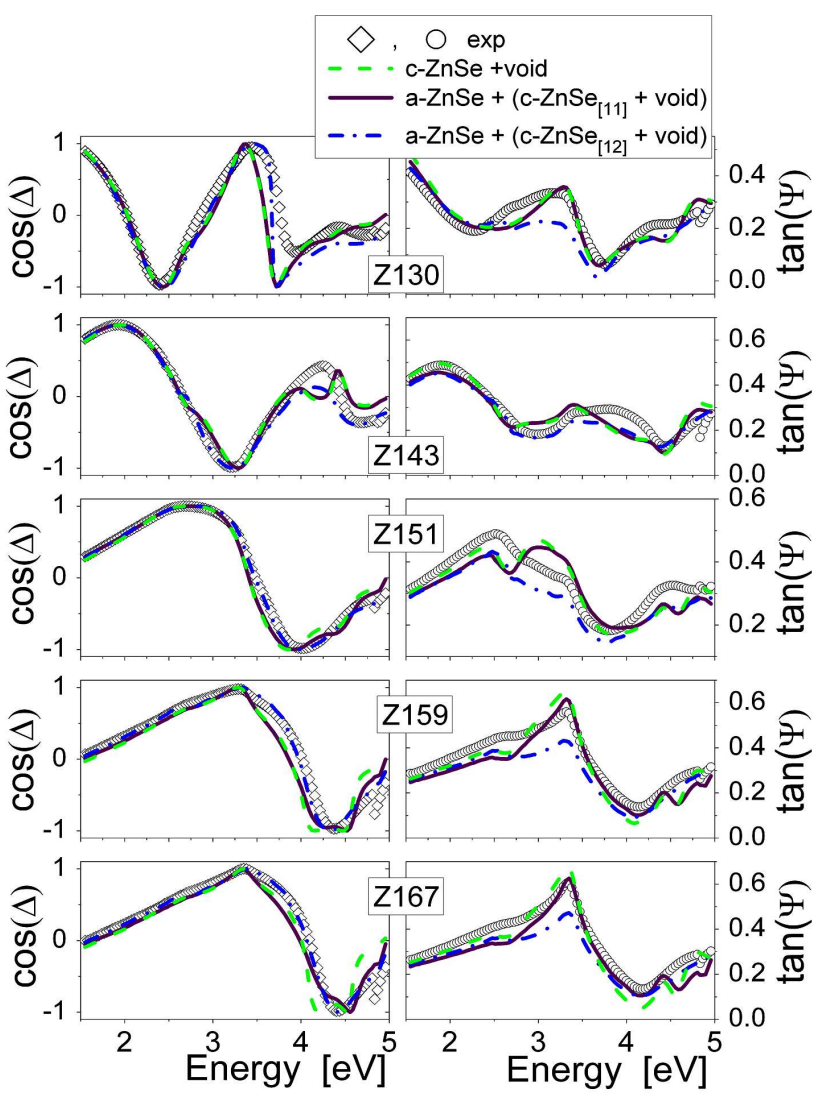

Fig. 4. The room-temperature ellipsometric data for $\cos (\Delta)$ and $\tan (\Psi)$ of ZnSe single layers (symbols). Dash, solid and dash-dot lines represent model generated curves obtained by considering nanolayers as porous c-ZnSe and two different Bruggeman effective medium mixtures of porous c-ZnSe and a-ZnSe, respectively.

The results of AFM and Raman scattering measurements, as well as our previous results of optical transmission measurements [10], strongly support this suggestion.

If materials are composed of phases with the size much smaller than the radiation wavelength, but large enough to retain their bulk properties, then the EMA may generally be used for modeling such structures. The self-consistent Bruggeman EMA (B-EMA) is the most frequently used approach ([6] and references therein). The average crystallite size in our ZnSe layers fulfills both of mentioned conditions: the crystallite is small enough in comparison to the radiation wavelength applied in the SE measurement, but still quite large to exclude more pronounced quantum size effects.

Creation of pits and increase of the surface roughness [8], due to relaxation of highly strained as-deposited ZnSe films with time, leads to increased porosity of ZnSe layers and necessity of treating the layers as porous material, described as a mixture of $\mathrm{ZnSe}$ and voids. Therefore, we investigated the possibility of modelling the layers as a Bruggeman effective-medium mixture of single- -crystalline ZnSe and voids, taking the crystalline ZnSe dielectric function from SOPRA database [11]. The best fit of SE data was attained by this model with volume fractions of voids about 0.17 for all layers (fitting curves presented in Fig. 4 by dashed lines). The single layer thicknesses estimated from such fitting procedure are shown in Fig. 5 by up-triangles, whereas the thicknesses expected according to the preparation procedure are represented by open circles. Let us note that the estimated and expected thicknesses are in reasonable agreement except for the thinnest layer, where discrepancy between these values is greater than $20 \%$. This is most probably the consequence of high disorder and presence of large amount of amorphous phase in $30 \mathrm{~nm}$ thick layer.

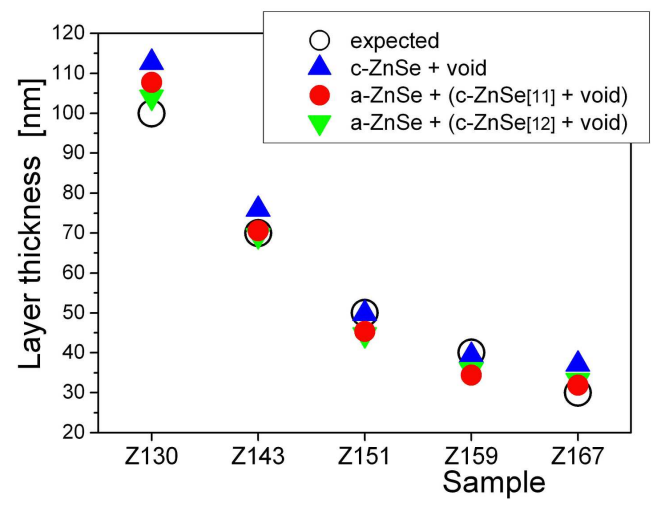

Fig. 5. Expected layer thickness of ZnSe films together with the values obtained by layer modelling with three different Bruggeman effective-medium mixture.

Afterwards, we considered the single layers as a large-grain polycrystalline material [6] modeled by Bruggeman effective-medium mixture of porous c-ZnSe, with volume fractions of voids about 0.17 , and amorphous ZnSe. The dielectric function of amorphous ZnSe was taken from single available literature data [9], whereas the dielectric function of c-ZnSe was assumed in two different forms. Fitting curves obtained using c-ZnSe dielectric function from SOPRA database [11] and from the other published data [12] are presented in Fig. 4 by solid and dash-dot lines, respectively. The single layer thicknesses estimated from such fitting procedures are shown in Fig. 5 by full circles and down-triangles, respectively. Although both results show acceptable agreement with the experimental data, we chose as final those with c-ZnSe dielectric function from SOPRA database, because they had somewhat better goodness of fit (i.e. higher values of coefficient of determination, $R^{2}$ ) for the whole series of investigated ZnSe films. In this approach, beside the layer thickness, the volume fraction of the amorphous component in each layer was treated as adjustable parameter. The results of such fitting procedure are presented in Fig. 6 for all investigated ZnSe films. It is obvious that a-ZnSe volume fraction drastically increases with decreasing layer thickness, which supports the assumption made according to the AFM results where short 


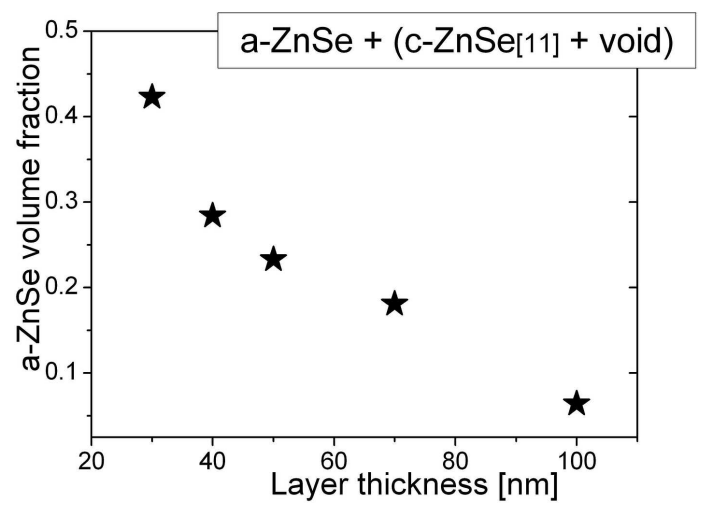

Fig. 6. Relationship between a-ZnSe volume fraction and the expected thickness of the layers treated as Bruggeman effective-medium mixture of porous c-ZnSe and a-ZnSe.

scale variations, related to crystal grains, were more frequently seen in the thicker layers.

\section{Conclusion}

The microstructure of a series of ZnSe single layers, having thicknesses between 30 and $100 \mathrm{~nm}$ deposited at room substrate temperature by thermal evaporation of $\mathrm{ZnSe}$ powder in vacuum is characterized by SE, AFM and Raman scattering. It is shown that experimental SE and model-generated results can reach reasonable agreement when ZnSe nanolayers are treated as polycrystalline material modeled by the Bruggeman effective medium mixture of porous c-ZnSe and a-ZnSe. This layer structure is fully consistent with the findings of AFM and Raman scattering measurements, as well as with our previous results of optical transmission measurements. The data obtained by SE also point out to an increase of the amorphous phase volume fraction with decreasing layer thickness, which confirms and quantitatively describes the assumption made according to AFM.

The study presented in this paper demonstrates that SE can give very useful and detailed information about crystallinity and nanostructure of semiconductor thin films.

\section{Acknowledgments}

This work is supported by the Serbian Ministry of Science under the Project No. 141047 and bilateral project between Bulgarian Academy of Sciences and Serbian Academy of Sciences and Arts.

\section{References}

[1] D. Nesheva, Z. Aneva, S. Reynolds, C. Main, A.G. Fitzgerald, J. Optoel. Adv. Mater. 8, 2120 (2006).

[2] H. Shoji, Y. Nakata, K. Mukai, Y. Sugiyama, M. Sugawara, N. Yokoyama, H. Ishikawa, Electron. Lett. 32, 2023 (1996).

[3] P. Reiss, G. Quemard, S. Carayon, J. Bleuse, F. Chandezon, A. Pron, Mater. Chem. Phys. 84, 103 (2004).

[4] D.S. Patil, D.K. Gautam, Physica B 344, 140 (2004).

[5] E. Guziewicz, M. Godlewski, K. Kopalko, E. Lusakowska, E. Dynowska, M. Guziewicz, M.M. Godlewski, M. Phillips, Thin Solid Films 446, 172 (2004).

[6] P. Petrik, Phys. Status Solidi A 205, 732 (2008).

[7] R.B. Kale, C.D. Lokhande, R.S. Mane, Sung-Hwan Han, Appl. Surf. Sci. 252, 5768 (2006).

[8] D. Nesheva, M.J. Šćepanović, S. Aškrabić, Z. Levi, I. Bineva, Z.V. Popović, Acta Phys. Pol. A 116, 75 (2009).

[9] H.-Y. Lee, S.-J. Kim, J.-W. Kim, H.-B. Chung, Thin Solid Films 441, 214 (2003).

[10] D. Nesheva, M.J. Šćepanović, Z. Levi, S. Aškrabić, Z. Aneva, A. Petrovac, Z.V. Popović, J. Optoel. Adv. Mater. 11, 1351 (2009).

[11] E.D. Palik, Handbook of Optical Constants of Solids, Academic Press, New York 1985.

[12] S. Adachi, T. Taguchi, Phys. Rev. B 43, 9569 (1991). 